but also improved as children got older $(F(1,226)=4 \cdot 43$, $P=0 \cdot 03, t=-1 \cdot 847$ ).

Conclusions: The present study adds novel data in that multiple domains of balance performance (balance stability, sensorimotor balance) were examined, suggesting that children within the 'normal weight' category exhibit superior balance scores compared with overweight/obese children.

\title{
36 - Patterns of physical activity in primary-school children: the effect of ethnicity
}

\author{
EL Eyre $^{1}$, E Smith $^{1}$, S Kumar ${ }^{1}$, AJ Wagenmakers ${ }^{2}$ and KA Matyka ${ }^{1}$ \\ ${ }^{1}$ Warwick Medical School, The University of Warwick, UK: ${ }^{2}$ Schools of Sport and Exercise Sciences, University of \\ Birmingham, UK
}

Introduction: Ethnicity is an important predictor of metabolic health and the reasons for this are likely to be multifactorial. Differences in physical activity (PA) may contribute to this risk but few data exists in childhood.

Method: 122 (57 White EU, 36 South Asian, 29 other ethnic) children (mean age 8.5 (SD 0.5) years) wore a combined physical activity/heart rate (Actiheart, UK) monitor for $7 \mathrm{~d}$.

Median daily activity counts per minute (CPM) were compared between ethnic groups using Wilcoxon signed-rank test.

Results: Examining the group as whole, PA is greater on weekdays than weekends $(109 v .99 \mathrm{cpm},=3.92$, $P=0 \cdot 000)$ and during school than after school (117 v. $99 \mathrm{cpm},=-3 \cdot 22, P=0 \cdot 001)$. Compared with children from all ethnic backgrounds, White EU were more active on weekdays $(u=1376,=-2 \cdot 45, \quad P=0 \cdot 014 ;$ mean rank $=70 v$. 54, white EU $v$. all other ethnic groups, respectively) and after school $(u=1237,=-3 \cdot 16, P=0 \cdot 002$, mean rank $=72 v$. 52). Subgroup analysis showed that South Asian children had no differences between weekday and weekend PA (103 v. $92 \mathrm{cpm},=1.654, P=0.098)$ but were more active at school than after school (122 v. 91, $=3 \cdot 174$, $P=0 \cdot 002)$. White EU children were more active on weekdays than weekends (116 v. $90 \mathrm{cpm},=-2 \cdot 24, P=0 \cdot 025)$ but did similar activity after school and during school (118 v. $112 \mathrm{cpm},=4 \cdot 65, P=0 \cdot 642)$.

Conclusions: Ethnic groups exercise differently but all children engage in highest activity at school. The contribution of PA on metabolic well-being needs further investigation in vulnerable groups of children.

\section{7 - South Asian children spend more time in light activities and less time in moderate and vigorous PA on weekdays}

\author{
ELJ Eyre ${ }^{1}$, S Kumar ${ }^{1}$, E Smith $^{1}$, AJ Wagenmakers ${ }^{2}$ and KA Matyka ${ }^{1}$ \\ ${ }^{1}$ Warwick Medical School, The University of Warwick, UK: ${ }^{2}$ School of Sport and Exercise Sciences, The University \\ of Birmingham, UK
}

Introduction: South Asian (SA) children are at increased metabolic risk compared with White children. The role of physical activity in this risk is unknown. The present study compares metabolic equivalent (MET) levels in SA and White EU children.

Method: Ninety-five (54 White EU, 41 SA) children (mean age $8.4(\mathrm{SD} 0 \cdot 5)$ years) wore a combined activity and heart rate monitor (Actiheart, Cambridge, UK) for $7 \mathrm{~d}$. Time spent in MET levels were categorized into light (LPA), moderate (MPA) and vigorous physical activity (VPA). Results were analysed using the Mann-Whitney test.

Results: SA children spent fewer minutes (average $7 \mathrm{~d}$ ) in VPA ( $U=589,=-4 \cdot 12, P=0 \cdot 000$, SA mean rank $=34 \cdot 62 v$. White EU mean rank $=39 \cdot 68)$ and more minutes in LPA than White $\mathrm{EU}(U=889,-1 \cdot 82, P=0.034$, SA mean rank $=54.37 v$. White EU 43.94). SA children spent fewer weekday minutes in MPA $(U=875,=-1 \cdot 75, P=0 \cdot 040$, mean rank $42 \cdot 33 v .52 \cdot 31 \mathrm{SA} v$. White EU, respectively) and 